\title{
Comparative Planetary Atmospheres of the Galilean Satellites
}

\author{
Darrell F. Strobel
}

Johns Hopkins University, Baltimore, MD 21218 USA, strobel@jhu.edu

We know that each of the Galilean satellites of Jupiter has a tenuous atmosphere by terrestrial standards. Io's $\mathrm{SO}_{2}$ equatorial atmosphere and, perhaps, Callisto's atmosphere inferred from its large ionospheric densities are measured in nanobars, whereas the atmospheres of Europa and Ganymede produced by ion sputtering of the water ice surfaces only reach picobar pressures and are comprised mostly of $\mathrm{O}_{2}$. Io's polar atmosphere is probably an order of magnitude less dense than its equatorial atmosphere. Europa and Ganymede have $\mathrm{O}_{2}$ atmospheres with column densities in the range of $(1-10) \times 10^{14} \mathrm{~cm}^{-2}$. These atmospheres, on the basis of their inferred production and loss rates, are estimated to have short residence times of $\sim 2-3$ days.

For Io and Europa, the harsh environment of Jupiter's inner magnetosphere produces atmospheric chemistry driven by magnetospheric electrons as well as by solar radiation. In fact, ionization and UV emission occur predominantly by electron impact. With the much lower magnetospheric electron densities at the orbits of Ganymede and Callisto solar photoionization exceeds electron impact ionization.

Based on measured ionospheric electron density profiles $\left(\sim 10^{4}-10^{5} \mathrm{~cm}^{-3}\right)$ for Io, Europa, and Callisto, the ionospheric Pedersen and Hall conductances can be computed to estimate the strengths of electrodynamic interactions of Io, Europa, and Callisto with the Io plasma torus (plasma in Jupiter's inner magnetosphere). As a result of these interactions large electric currents flow through their ionospheres $\left(\sim 10^{6} \mathrm{~A}\right)$ accompanied by large Joule heating rates that are the dominant heating mechanism of their atmospheres, leading to estimates of high temperatures $\sim 1000-2000 \mathrm{~K}$ for altitudes 2 scale heights above their surfaces. The magnetospheric plasma interaction with these atmospheres can be remotely sensed by HST/STIS observations. The regions of brightest UV line emissions from OI (and SI on Io) indicate the regions of maximum deposition of magnetospheric electron power. On Io, this UV auroral emission occurs in equatorial spots organized by Jupiter's magnetospheric field and brightest where the field is closest to the surface, but above the limb. Theoretical models can explain why UV emission is preferentially brighter at the equator than the poles. On Europa with a thinner atmosphere, the UV emission is primarily limb glow with one bright region on the disk. The latter is not understood.

In the following table, various quantities for the Galilean satellites are summarized. For the electrodynamic interactions, the relevant quantities are the ionospheric Pedersen conductance, $\Sigma_{\mathrm{Ped}}$, the Alfven conductance, $\Sigma_{\mathrm{A}}$, the velocity of magnetospheric plasma relative to the satellite, $V_{\text {rel plasma }}$, the Alfven velocity, $\mathrm{V}_{\text {alfven }}$, the Alfven Mach number, $\mathrm{M}_{A}$, the sound speed Mach number, $\mathrm{M}_{S}$, the magnetic field, $\mathrm{B}$, the corotation electric field, $\mathrm{E}_{0}$, the ratio of the 
ionospheric electric field to the corotation electric field, $\alpha$, the total ionospheric electric current, $\mathrm{I}_{\mathrm{iono}}$, and the perturbation magnetic field due to ionospheric currents relative to the background field, $\left|\Delta \mathrm{B} / \mathrm{B}_{J}\right|$.

\begin{tabular}{|c|c|c|c|c|}
\hline Quantity & $\mathrm{IO}$ & EUROPA & GANYMEDE & CALLISTO \\
\hline $\begin{array}{l}\text { Atmospheric } \\
\text { source }\end{array}$ & Volcanos & $\begin{array}{l}\text { Sputtering } \\
\mathrm{H}_{2} \mathrm{O} \text { Ice } \\
\end{array}$ & $\begin{array}{l}\text { Sputtering } \\
\mathrm{H}_{2} \mathrm{O} \text { Ice } \\
\end{array}$ & $\begin{array}{l}\text { Sputtering } \\
\mathrm{H}_{2} \mathrm{O} \text { Ice }\end{array}$ \\
\hline $\begin{array}{l}\text { Column Density } \\
\left(\mathrm{cm}^{-3}\right)\end{array}$ & $\begin{array}{l}2 \times 10^{16} \\
(0.03-3) \\
\times 10^{16}\end{array}$ & $\begin{array}{l}5 \times 10^{14} \\
(3-7) \times 10^{14}\end{array}$ & $\begin{array}{l}5 \times 10^{14} \\
(1-10) \times 10^{14}\end{array}$ & $\begin{array}{l}8 \times 10^{14} \mathrm{CO}_{2} \\
\sim 10^{16} \mathrm{O}_{2}\end{array}$ \\
\hline Major Gas & $\mathrm{SO}_{2}$ & $\mathrm{O}_{2}$ & $\mathrm{O}_{2}$ & $\mathrm{O}_{2}$ or $\mathrm{CO}_{2}$ ? \\
\hline Other Species & $\begin{array}{l}\mathrm{S}_{2}, \mathrm{SO}, \mathrm{O}, \mathrm{S} \\
\mathrm{NaCl}, \mathrm{Na}, \mathrm{K}\end{array}$ & $\begin{array}{l}\mathrm{O}, \mathrm{H}_{2} \mathrm{O}, \mathrm{H}_{2}, \\
\mathrm{H}, \mathrm{Na}, \mathrm{K}\end{array}$ & $\begin{array}{l}\mathrm{O}, \mathrm{H}_{2} \mathrm{O} \\
\mathrm{H}_{2}, \mathrm{H}\end{array}$ & $\begin{array}{l}\mathrm{CO}_{2}, \mathrm{O}, \mathrm{H}_{2} \mathrm{O}, \\
\mathrm{H}_{2}, \mathrm{H}, \mathrm{CO}, \mathrm{C}\end{array}$ \\
\hline $\begin{array}{l}\text { Thermospheric } \\
\text { Temperature (K) }\end{array}$ & $\sim 2000$ & $\sim 2000$ & $\sim 1000$ & $\sim 2000$ \\
\hline Escape rate $\left(\mathrm{s}^{-1}\right)$ & $\begin{array}{l}1.6 \times 10^{28} \\
\mathrm{SO}_{2}\end{array}$ & $1 \times 10^{27} \mathrm{O}_{2}$ & $?$ & $?$ \\
\hline Lifetime (d) & 3 & 2 & & \\
\hline $\begin{array}{l}\text { Peak Electron } \\
\text { Density }\left(\mathrm{cm}^{-3}\right)\end{array}$ & $\sim 3 \times 10^{5}$ & $\sim 10^{4}$ & $<10^{3}$ & $\sim 2 \times 10^{4}$ \\
\hline$\Sigma_{\text {Ped }}(\mathrm{mho})$ & $130(\mathrm{ec})$ & 14 & $\leq 100(\mathrm{pu})$ & $\sim 10^{4}(\mathrm{ec})$ \\
\hline$\Sigma_{\mathrm{A}}(\mathrm{mho})$ & 5 & 1.7 & 2 & 1.3 \\
\hline $\begin{array}{l}V_{\text {rel plasma }} \\
\left(\mathrm{km} \mathrm{s}^{-1}\right)\end{array}$ & 57 & 90 & 180 & 170 \\
\hline$V_{\text {alfven }}\left(\mathrm{km} \mathrm{s}^{-1}\right)$ & 220 & 460 & 375 & 600 \\
\hline $\mathrm{M}_{A}$ & 0.25 & 0.2 & 0.5 & 0.3 \\
\hline $\mathrm{M}_{S}$ & 1.65 & 1.75 & 2.4 & 2.4 \\
\hline $\mathrm{B}(\mathrm{nT})$ & $1800, \mathrm{~J}$ & $420, \mathrm{~J}$ & $750, \mathrm{G}$ & $35, \mathrm{~J}$ \\
\hline $\mathrm{E}_{0}(\mathrm{Vm}-1)$ & 0.1 & 0.04 & 0.02 & 0.006 \\
\hline$\alpha=\mathrm{E}_{i} / \mathrm{E}_{0}$ & 0.1 & 0.2 & - & 0.001 \\
\hline $\mathrm{I}_{\text {iono }}\left(10^{6} \mathrm{~A}\right)$ & 10 & 1.4 & - & 0.15 \\
\hline $\begin{array}{l}\text { Joule Heating } \\
\text { (W) }\end{array}$ & $4 \times 10^{11}$ & $3 \times 10^{10}$ & $?$ & $\leq 10^{9}$ \\
\hline$\left|\Delta \mathrm{B} / \mathrm{B}_{J}\right|$ & 0.45 & 0.4 & - & 0.35 \\
\hline $\begin{array}{l}\text { Magnetospheric } \\
\text { Thermal Ion } \\
\text { Power (W) }\end{array}$ & $2 \times 10^{9}$ & $1 \times 10^{8}$ & $\sim 10^{7}$ & $\sim 10^{4}$ \\
\hline $\begin{array}{l}\text { Solar EUV/UV } \\
\text { Power Input (W) }\end{array}$ & $7 \times 10^{9}$ & $<10^{8}$ & $<10^{8}$ & $5 \times 10^{7}, \mathrm{CO}_{2}$ \\
\hline
\end{tabular}

$\mathrm{ec}=$ elastic collisions, $\mathrm{pu}=$ pickup, $\mathrm{J}=$ Jupiter's magnetic field, $\mathrm{G}=$ Ganymede's magnetic field 